\title{
Calculation and Analysis of the Inductance Gradient of Serial-stacked Enhanced Railguns
}

\author{
Z.M. QIAO, B. LEI, Q.A. LV \& Y.C. XING \\ Shijiazhuang Mechanical Engineering College, Shijiazhuang, China
}

\begin{abstract}
Serial-stacked enhanced railguns are with bigger inductance gradient value than simple ones, which is important to improving the energy efficiency and the railguns' launch force. On the basis that the structure and principles of serial-stacked enhanced railguns are introduced. We calculate the inductance gradient of the enhanced railguns at low frequency. The result is that with a fixed caliber, the inductance gradient value increases as the gap of rails increases, and it decreases as width of the rails increases. On the condition that the caliber varies with structure parameters of rails by certain rules, the inductance gradient value increases as the gap of rails increases, and it increases as thickness of the rails increases, as well. The analysis lays the foundation for the serial-stacked enhanced railguns' design, which is of certain value to the railguns' application.
\end{abstract}

KEYWORD: serial-stacked enhanced railguns; inductance gradient; structural parameters

\section{INTRODUCTION}

Electromagnetic railguns can launch armatures and projectiles to an ultra high-speed with Ampere force, which is an important application of electromagnetic energy in the military. The railguns can be used for rapid strike, air defense, anti-missile and other fields for the advantage of high-speed launch. So it generates the interest of many researchers, and it has been the current research focus of many the major powers of the world, such as the USA and Russia.

Inductance gradient value is an important parameter for railguns, because the launch force is proportional to the inductance gradient value under similar conditions, and increasing the inductance gradient value also means more energy of the pulse power acting on the guns' structure, reducing unnecessary energy losses in the power supply and connecting cables. It has a great value for improving guns' performance and energy efficiency. The inductance gradient of traditional simple railguns with large-caliber is small, which is about $0.5 \mu \mathrm{H} / \mathrm{m}$ or so. The electromagnetic force is not high enough under limited current input. So there is disadvantageous position in application of the large muzzle energy guns. The enhanced electromagnetic railguns technology has become a hot topic in the field of electromagnetic launch. The enhanced railguns adopt a flat or stacked multi-turn form, so the magnetic field generated is greatly improved. It can produce a strong magnetic force to the armature and projectiles by using a lower current. It is proposed in 16th international conference on electromagnetic launch that the inductance gradient value can be greatly increased by using serial-stacked railguns. By changing the contact mode, serial-stacked enhanced railguns uses two multi-turn structure similar as coils. This structure can improve railgun inductance gradient, and enhance the electromagnetic force. It also makes the single rail thin, which is good to a more uniform current distribution.

For simple railguns, inductance gradient calculation method is existed, which plays an important role in simple railguns' design, and is significant to railguns' analysis. But the serial-stacked railguns' design theory is still in the exploratory stage, and value and the relationship of the structure and the inductance has not been explored and researched indepth. We assumes uniform current distribution in the track, and gives a calculation method for the serial-stacked enhanced railguns. We analyze the relationship of the structure and the inductance of it. This paper promotes engineering research for serialstacked railguns and have a good reference for their design.

\section{STRUCTURE AND PRINCIPLES OF SERIAL- STACKED ENHANCED RAILGUNS}

The structure of the serial-stacked enhanced railgun is shown in Figure 1. (a). The structure consist of 
two terms of rails which are similar with multi-turn coils and muti-turn armature which contacts with the rails. The different layers of rails and armature are insulated from each other. The two rail terms are placed in parallel as the guns' body. It is clearly seen how the railgun works in Figure 1. (b). From a single set of armature / rail vice, the direction of the current, magnetic direction of the guns' bore and the magnetic force's direction on the armature are vertical with one another according to Ampere Role. Because the current runs in the multiple rails and armature with 3 cycles, it causes much greater magnetic field strength than a simple single-turn railguns' structure does under the same condition. The right side of the rails generates a similar magnetic field with the left mirror image, and they are symmetrical, which further enhances the electromagnetic force, and promote the armature to high-speed.

Based on Law of Conservation of Energy and virtual work theorem, we can calculate the electromagnetic launch force $F$ :

$F=\frac{1}{2} L^{\prime} I^{2}$

And $L$ ' stands for the inductance gradient of serial-stacked enhanced railguns and $I$ is the current input.

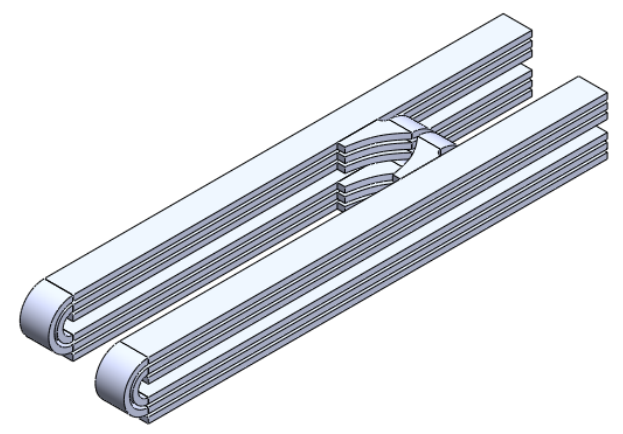

(a) The structure of the serial-stacked enhanced railgun

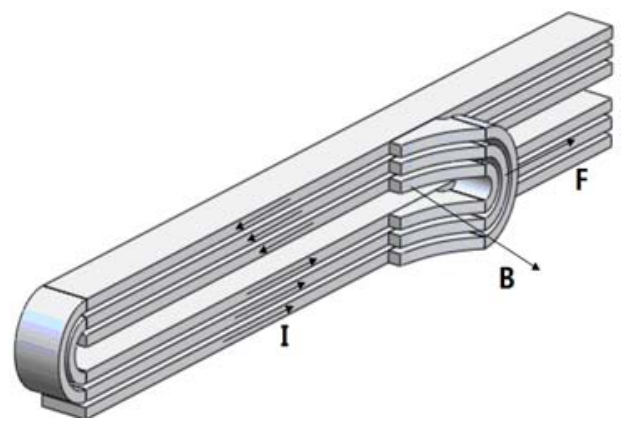

(b) Principles of the serial-stacked enhanced railgun

Figure 1. The structure and principles of the serial-stacked enhanced railgun

\section{CALCULATION METHOD}

\subsection{Basic structural parameters}

Since in the railgun stucture, the longitudinal dimension is much larger than the cross-sectional dimension, the calculation method we choose is the current line element method, the cross-sectional dimension of the rails is shown in Figure 2. It can be divided into four sets of rails as upper left, lower left, upper right, lower right. The thickness of the gaps in a set is $d(\mathrm{~mm})$, the gap between the upper and lower rail group is $b(\mathrm{~mm})$ (to meet the processing technology, $b$ is numerically equal to $c+2 d)$. The sectional dimension of a single rail is $e(\mathrm{~mm}) \times c(\mathrm{~mm})$. The gap between the left and right track group is $a(\mathrm{~mm})$ ( $a$ is equal to $b+6 c+4 d)$, which means between the left and right rail groups, it forms $a(\mathrm{~mm}) \times a(\mathrm{~mm})$ square bore.

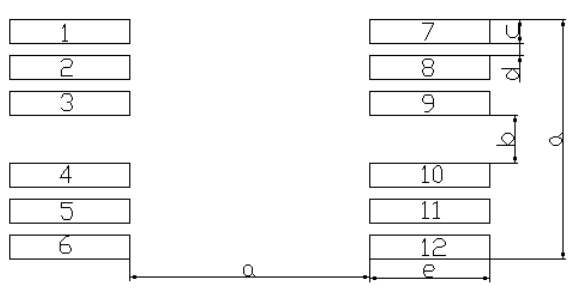

Figure 2. Structural parameters and the cross-sectional dimension of the rails

\subsection{Calculation method}

For a linear inductance calculation, the general formula is:

$L=N-G$

Where, $N$ is related with the shape and size of the rails. $G, A$ and $Q$ is related with the wire's crosssectional shape and the size of the current distribution on the section, and under normal circumstances, $A-Q$ is much smaller than $N-G$, so it can be always negligible.

So for most linear inductance calculation, the formula can be:

$L=N-G$

For the reason that the low frequency inductance gradient of railguns is always used in the estimation of the railguns' performance before their design. We calculate the inductance calculations according to formula (3) as well. We suppose that the current flows through the rail evenly, and calculate the $N$ depending on the assumptions that current is a line by using the geometrical distance method. We calculate the $G$ value by the way to calculate mutual inductance between the respective current lines. Therefore, we can calculate and give the inductance $L_{i}$ for a single rail $i$, and the mutual inductance $M_{i k}$ between the two rails $i, k$ :

$N=\frac{\mu_{0} l}{2 \pi}(\ln (2 l)-1)$ 
$G=\frac{\mu_{0} l}{2 \pi} \ln g$

$L_{i}=\frac{\mu_{0} l}{2 \pi}\left(\ln \left(\frac{l}{g}\right)-1\right)$

$M_{i k}=\frac{\mu_{0} l}{2 \pi}\left(\ln \left(\frac{2 l}{g_{i k}}\right)-1\right)$

Where, $l$ is the rail length, $g$ is the mean geometrical distance of the cross section of the conductor. Therefore, by use of the mean geometrical distance method, we can solve the inductance value. And for areas $i$ and $k$, the geometric mean distance value $g$ can be calculated as:

$\ln g=\frac{1}{s_{i} s_{k}} \int_{s_{i}} \int_{s_{k}} \ln \eta d s_{i} d s_{k}$

For the serial-stacked enhanced railguns system, there are a total of twelve rails. And for a linear system composed of $n$ rails, the inductance value $L$ is:

$$
L=\sum_{i=1}^{n} L_{i}+2 \sum_{j=1}^{n} \sum_{k=1}^{n} M_{j k}
$$

\section{RESULTS}

\section{1 $M$ ' or $L$ ' values of rails of a certain structure}

On the condition that $\mathrm{a}=100 \mathrm{~mm}, \mathrm{~b}=20 \mathrm{~mm}, \mathrm{c}=$ $10 \mathrm{~mm}, \mathrm{~d}=5 \mathrm{~mm} \& \mathrm{e}=50 \mathrm{~mm}$. According to their number 1-12 in figure 1, The mutual inductance gradient $M^{\prime}$ of every two rails and inductance gradient $L$ ' of every rails are listed in Table1. the first row and the first column are rail number, and the other value are $M^{\prime}$ or $L$ '. For example, 4.0041 in the second row and the second column represents that $L^{\prime}$ of number 1 rail is $4.0041 \mu \mathrm{H} / \mathrm{m}$, and 3.4981 in the second row and the third column represents that $M^{\prime}$ ' of number1 rail and number 2 rail is $3.4981 \mu \mathrm{H} / \mathrm{m}$. Finally, the system inductance gradient is $8.2638 \mu$ $\mathrm{H} / \mathrm{m}$.

Table 1 The mutual inductance gradient M' of every two rails and inductance gradient L' of every rails

\begin{tabular}{rccccccccccccc}
\hline & 1 & 2 & 3 & 4 & 5 & 6 & 7 & 8 & 9 & 10 & 11 \\
\hline 1 & 4.0041 & 3.4981 & 3.0415 & -2.4579 & -2.2517 & -2.0789 & 1.5975 & 1.5945 & 1.5815 & -1.5235 & -1.483 & -1.4379 \\
2 & 3.4981 & 4.0041 & 3.4981 & -2.7124 & -2.4579 & -2.2517 & 1.5945 & 1.5975 & 1.5945 & -1.5572 & -1.5235 & -1.483 \\
3 & 3.0415 & 3.4981 & 4.0041 & -3.0415 & -2.7124 & -2.4579 & 1.5815 & 1.5945 & 1.5975 & -1.5815 & -1.5572 & -1.5235 \\
4 & -2.4579 & -2.7124 & -3.0415 & 4.0041 & 3.4981 & 3.0415 & -1.5235 & -1.5572 & -1.5815 & 1.5975 & 1.5945 & 1.5815 \\
5 & -2.2517 & -2.4579 & -2.7124 & 3.4981 & 4.0041 & 3.4981 & -1.483 & -1.5235 & -1.5572 & 1.5945 & 1.5975 & 1.5945 \\
6 & -2.0789 & -2.2517 & -2.4579 & 3.0415 & 3.4981 & 4.0041 & -1.4379 & -1.483 & -1.5235 & 1.5815 & 1.5945 & 1.5975 \\
7 & 1.5975 & 1.5945 & 1.5815 & -1.5235 & -1.483 & -1.4379 & 4.0041 & 3.4981 & 3.0415 & -2.4579 & -2.2517 & -2.0789 \\
8 & 1.5945 & 1.5975 & 1.5945 & -1.5572 & -1.5235 & -1.483 & 3.4981 & 4.0041 & 3.4981 & -2.7124 & -2.4579 & -2.2517 \\
9 & 1.5815 & 1.5945 & 1.5975 & -1.5815 & -1.5572 & -1.5235 & 3.0415 & 3.4981 & 4.0041 & -3.0415 & -2.7124 & -2.4579 \\
10 & -1.5235 & -1.5572 & -1.5815 & 1.5975 & 1.5945 & 1.5815 & -2.4579 & -2.7124 & -3.0415 & 4.0041 & 3.4981 & 3.0415 \\
11 & -1.483 & -1.5235 & -1.5572 & 1.5945 & 1.5975 & 1.5945 & -2.2517 & -2.4579 & -2.7124 & 3.4981 & 4.0041 & 3.4981 \\
12 & -1.4379 & -1.483 & -1.5235 & 1.5815 & 1.5945 & 1.5975 & -2.0789 & -2.2517 & -2.4579 & 3.0415 & 3.4981 & 4.0041 \\
\hline
\end{tabular}

\section{2 the system inductance gradient L' of different structural parameters}

On the condition that $a=100 \mathrm{~mm}, b=20 \mathrm{~mm} \& e=$ $50 \mathrm{~mm}$. which means a fixed caliber. The gap of rails in a group $d$ and rails' thickness $c$ are seen as variables, and they satisfy the rule ' $c+d=15 \mathrm{~mm}$ '. The relation between the system inductance gradient $L$, and the gap $d$ is shown in Figure 3. According to the relationship, the inductance gradient value increases as the gap value $d$ increases. When the gap $d$ is $4 \mathrm{~mm}$, the inductance gradient value is $8.23 \mu \mathrm{H} / \mathrm{m}$, and when the gap $d$ is $10 \mathrm{~mm}$, the inductance gradient value is $8.46 \mu \mathrm{H} / \mathrm{m}$

On the condition that $a=100 \mathrm{~mm}, b=20 \mathrm{~mm}, c=$ $10 \mathrm{~mm} \& d=5 \mathrm{~mm}$. which means a fixed caliber of $100 \mathrm{~mm} \times 100 \mathrm{~mm}$, as well. The relation between the system inductance gradient $L$ ' and the rails' width e is shown in Figure 4. It can be seen that the induct- ance gradient value decreases as rails' width value $d$ increases. When the rails' width $e$ is $40 \mathrm{~mm}$, the inductance gradient value is $9.00 \mu \mathrm{H} / \mathrm{m}$, and when the rails' width $e$ is $10 \mathrm{~mm}$, the inductance gradient value is $5.86 \mu \mathrm{H} / \mathrm{m}$.

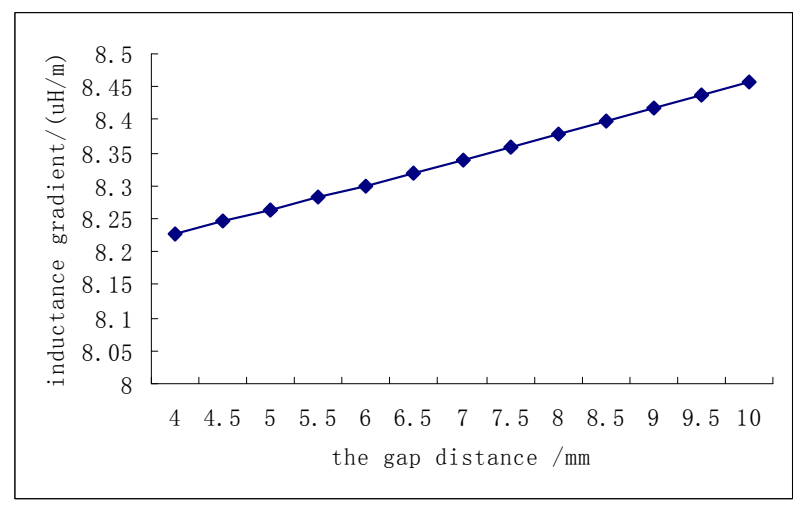

Figure 3. The relation between the system inductance gradient $L$ ' and the gap $d$ for a fixed $100 \mathrm{~mm} \times 100 \mathrm{~mm}$ caliber 


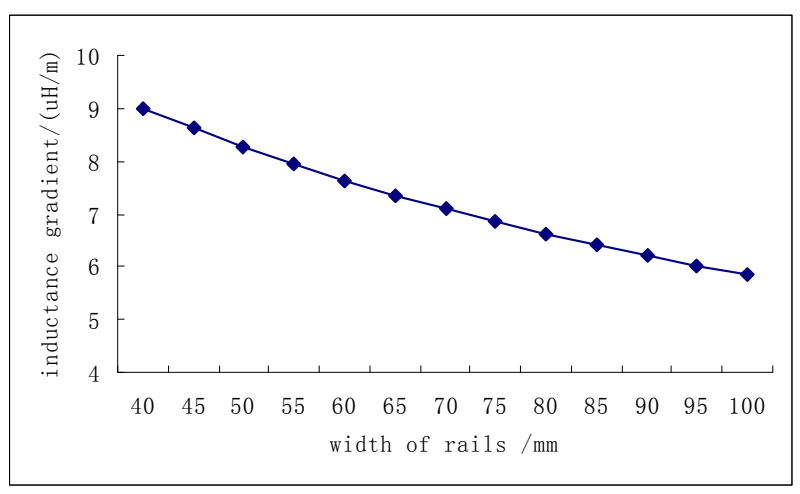

Figure 4. The relation between the system inductance gradient $L$ ' and the rail's width $e$ for a fixed $100 \mathrm{~mm} \times 100 \mathrm{~mm}$ caliber

On the condition that $e=50 \mathrm{~mm} \& c=10 \mathrm{~mm}$. Other structural parameters are seen as variables, and they satisfy the rule " $b=c+2 d, a=b+4 d+$ 6 ". The relation between the system inductance gradient $L$ ' and the gap $d$ is shown in Figure 5. According to the relationship, the inductance gradient value increases as the gap value $d$ increases. When the gap $d$ is $4 \mathrm{~mm}$, the inductance gradient value is $7.96 \mu \mathrm{H} / \mathrm{m}$, and when the gap $d$ is $10 \mathrm{~mm}$, the inductance gradient value is $9.01 \mu \mathrm{H} / \mathrm{m}$.

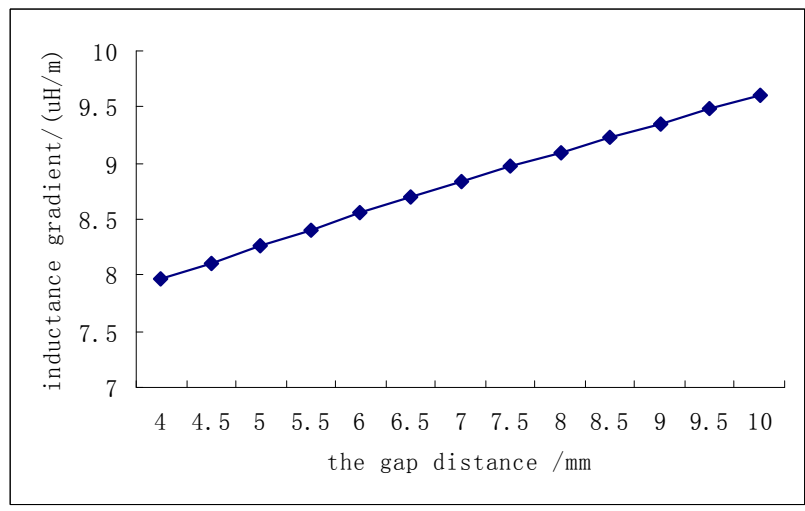

Figure 5 The relation between the system inductance gradient $L$ ' and the gap $d$ on the condition that the caliber is not fixed

On the condition that $e=50 \mathrm{~mm} \& d=5 \mathrm{~mm}$. Other structural parameters are seen as variables, and they satisfy the rule " $b=c+2 d, a=b+4 d+$ $6 c$ ". The relation between the system inductance gradient $L^{\prime}$ and the rail's thickness $c$ is shown in Figure 6. According to the relationship, the inductance gradient value increases as the rail's thickness $C$ increases. When the rail's thickness $c$ is $5 \mathrm{~mm}$, the inductance gradient value is $6.75 \mu \mathrm{H} / \mathrm{m}$, and when the rail's thickness $c$ is $15 \mathrm{~mm}$, the inductance gradient value is $9.43 \mu \mathrm{H} / \mathrm{m}$.

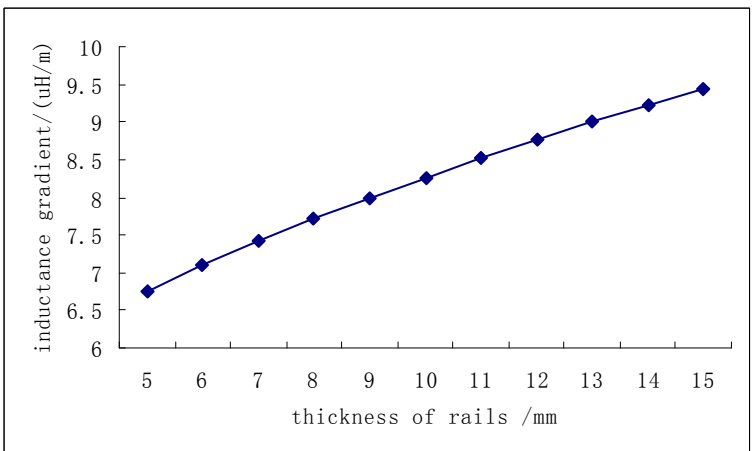

Figure 6. The relation between the system inductance gradient $L$ ' and the rail's thickness $c$ on the condition that the caliber is not fixed

\section{CONCLUSION}

Based on the structure and principle of serialstacked enhanced railguns, the calculation method is given, and the inductance gradient of serial-stacked enhanced railguns with different structural parameters are calculated and analyzed. On the fixed caliber conditions, the inductance gradient value increases as the gap value $d$ increases, and the inductance gradient value decreases as rails' width value $d$ increases. On the condition that the caliber is seen as variable, the inductance gradient value increases as the gap value $d$ increases, and the inductance gradient value increases as the rail's thickness $c$ increases, as well. The inductance gradient of serial-stacked enhanced railguns is much bigger than that of simple guns. The analysis lays the foundation for the serialstacked enhanced railguns' design.

\section{REFERENCES}

FAIR, H D. 1997. Electromagnetic launch: a review of the U.S. national program. IEEE Transactions on Magnetics, 33(1): 11-16.

LI, J. 2014. Electromagnetic gun technology and its Development. High Voltage Engineering 40(4): 1052-1064.

LV, Q. A.. 2013. Primary structural design and optimal armature simulation for a practical electromagnetic launcher. IEEE Trans. Plasma Sci., 41(5): 1403-1409.

LV, Q. A.. 2015. Physical principle and relevant restraining methods about velocity skin effect. IEEE Trans. Plasma Sci., 43(5): 1523-1529.

MCNAB, I. R. 2001. Parameters for an electromagnetic naval railgun. IEEE Transactions on Magnetics, 37(1): 223-228.

POLTANOV, A. E. 2001. Multi-turn railguns: concept analysis and experimental results. IEEE Transactions on Magnetics, 37(1): 457-461. 